\title{
A new explicit iteration method for variational inequalities on the set of common fixed points for a finite family of nonexpansive mappings
}

Jong Kyu Kim ${ }^{*}$ and Nguyen Buong ${ }^{2}$

${ }^{*}$ Correspondence:

jongkyuk@kyungnam.ac.kr 'Department of Mathematics

Education, Kyungnam University, Changwon, 631-701, Korea

Full list of author information is

available at the end of the article

\begin{abstract}
In this paper, we introduce a new explicit iteration method based on the steepest descent method and Krasnoselskii-Mann type method for finding a solution of a variational inequality involving a Lipschitz continuous and strongly monotone mapping on the set of common fixed points for a finite family of nonexpansive mappings in a real Hilbert space.

MSC: $41 \mathrm{~A} 65 ; 47 \mathrm{H} 17 ; 47 \mathrm{H} 20$
\end{abstract}

Keywords: contraction; common fixed points; hybrid steepest descent method; nonexpansive mappings; monotone mappings

\section{Introduction and preliminaries}

Let $C$ be a nonempty closed and convex subset of a real Hilbert space $H$ with the inner product $\langle\cdot, \cdot\rangle$ and the norm $\|\cdot\|$, and let $F: H \rightarrow H$ be a nonlinear mapping. The variational inequality problem is to find a point $p^{*} \in C$ such that

$$
\left\langle F\left(p^{*}\right), p-p^{*}\right\rangle \geq 0, \quad \forall p \in C .
$$

Variational inequalities were initially studied by Kinderlehrer and Stampacchia in [1], and since then have been widely investigated. They cover partial differential equations, optimal control, optimization, mathematical programming, mechanics, and finance (see [1-3]).

It is well known that if $F$ is an $L$-Lipschitz continuous and $\eta$-strongly monotone, i.e., $F$ satisfies the following conditions:

$$
\begin{aligned}
& \|F(x)-F(y)\| \leq L\|x-y\|, \\
& \langle F(x)-F(y), x-y\rangle \geq \eta\|x-y\|^{2},
\end{aligned}
$$

where $L$ and $\eta$ are fixed positive numbers, then (1.1) has a unique solution. It is also known that (1.1) is equivalent to the fixed point equation

$$
p=P_{C}(p-\mu F(p))
$$

๑ 2013 Kim and Buong; licensee Springer. This is an Open Access article distributed under the terms of the Creative Commons Attribution License (http://creativecommons.org/licenses/by/2.0), which permits unrestricted use, distribution, and reproduction in any medium, provided the original work is properly cited. 
where $P_{C}$ denotes the metric projection from $x \in H$ onto $C$ and $\mu$ is an arbitrarily positive constant.

The fixed point formulation (1.2) involves the metric projection $P_{C}$. To overcome the complexity caused by $P_{C}$, Yamada [4] introduced a hybrid steepest descent method for solving (1.1). His idea is stated as follows. Assume that $C=\bigcap_{i=1}^{N} \operatorname{Fix}\left(T_{i}\right)$, the set of common fixed points of a finite family of nonexpansive mappings $T_{i}$ on $H$ with an integer $N \geq 1$.

Recall that $T: H \rightarrow H$ is nonexpansive if

$$
\|T x-T y\| \leq\|x-y\|, \quad \forall x, y \in H
$$

and

$$
\operatorname{Fix}(T)=\{x \in H: x=T x\}
$$

denotes the fixed point set of $T$. Yamada proposed the following algorithm in [4]

$$
u_{k+1}=T_{[k+1]} u_{k}-\lambda_{k+1} \mu F\left(T_{[k+1]} u_{k}\right) \text {, }
$$

where $T_{[n]}=T_{n \bmod N}$, for integer $n \geq 1$, with the mod-function taking values in the set $\{1,2, \ldots, N\}, \mu \in\left(0,2 \eta / L^{2}\right)$ and $\left\{\lambda_{k}\right\} \subset(0,1)$, and proved that the sequence $\left\{u_{k}\right\}$ in (1.3) converges strongly to $p^{*}$ under the following conditions:

(L1) $\lim \lambda_{k}=0$;

(L2) $\sum \lambda_{k}=\infty$;

(L3) $\sum\left|\lambda_{k}-\lambda_{k+N}\right|<\infty$.

Further, Zeng and Yao [5] proved the same result with (L3) replaced by

(L4) $\lim \left(\lambda_{k}-\lambda_{k+N}\right) / \lambda_{k+N}=0$.

Theorem 1.1 [5] Let $H$ be a real Hilbert space, and let $F: H \rightarrow H$ be an L-Lipschitz continuous and $\eta$-strongly monotone mapping for some constants $L, \eta>0$. Let $\left\{T_{i}\right\}_{i=1}^{N}$ be $N$ nonexpansive self-maps of $H$ such that $C=\bigcap_{i=1}^{N} \operatorname{Fix}\left(T_{i}\right) \neq \emptyset, \mu \in\left(0,2 \eta / L^{2}\right)$, and let conditions (L1), (L2), (L4) be satisfied. Assume in addition that

$$
\begin{aligned}
C & =\bigcap_{i=1}^{N} \operatorname{Fix}\left(T_{i}\right)=\operatorname{Fix}\left(T_{1} T_{2} \cdots T_{N}\right) \\
& =\operatorname{Fix}\left(T_{N} T_{1} T_{2} \cdots T_{N-1}\right) \\
& =\cdots \\
& =\operatorname{Fix}\left(T_{2} T_{3} \cdots T_{N} T_{1}\right) .
\end{aligned}
$$

Then the sequence $\left\{u_{k}\right\}$ defined by (1.3) converges strongly to the unique element $p^{*}$ in (1.1).

It is not difficult to show that (L3) implies (L4) if $\lim \lambda_{k} / \lambda_{k+N}$ exists. However, in general, conditions (L3) and (L4) are not comparable, i.e., neither one of them implies the other (see [6] for details).

Recently, Zeng et al. [7] proposed the following iterative scheme:

$$
u_{k+1}=T_{[k+1]} u_{k}-\lambda_{k+1} \mu_{k+1} F\left(T_{[k+1]} u_{k}\right) \text {, }
$$

and proved the following result. 
Theorem 1.2 [7] Let $H$ be a real Hilbert space, and let $F: H \rightarrow H$ be an L-Lipschitz continuous and $\eta$-strongly monotone mapping for some constants $L, \eta>0$. Let $\left\{T_{i}\right\}_{i=1}^{N}$ be $N$ nonexpansive self-maps of $H$ such that

$$
C=\bigcap_{i=1}^{N} \operatorname{Fix}\left(T_{i}\right) \neq \emptyset
$$

and let $\mu_{k} \in\left(0,2 \eta / L^{2}\right)$. Assume that the following conditions hold:

(i) $\sum \lambda_{k}=\infty$, where $\left\{\lambda_{k}\right\} \subset(0,1)$;

(ii) $\left|\mu_{k}-\eta / L^{2}\right| \leq \sqrt{\eta^{2}-c L^{2}} / L^{2}$ for some $c \in\left(0, \eta^{2} / L^{2}\right)$;

(iii) $\lim \left(\mu_{k+N}-\left(\lambda_{k} / \lambda_{k+N}\right) \mu_{k}\right)=0$.

Assume in addition that (1.4) holds. If

$$
\limsup _{k \rightarrow \infty}\left\langle T_{[k+N]} \cdots T_{[k+1]} u_{k}-u_{k+N}, T_{[k+N]} \cdots T_{[k+1]} u_{k}-u_{k}\right\rangle \leq 0,
$$

then the sequence $\left\{u_{k}\right\}$ defined by (1.5) converges strongly to the unique element $p^{*}$ in (1.1).

They also showed that conditions (L1), (L2) and (L4) are sufficient for $\left\{u_{k}\right\}$ to be bounded and

$$
\lim _{k \rightarrow \infty}\left\|u_{k}-T_{[k+N]} \cdots T_{[k+1]} u_{k}\right\|=0
$$

So, (1.6) is satisfied. They did not give another sufficient condition different from (L1), (L2) and (L4).

Let $F x=A x-u$, where $A$ is a self-adjoint bounded linear mapping such that $A$ is strongly positive, i.e.,

$$
\langle A x, x\rangle \geq \eta\|x\|^{2}, \quad \forall x \in H
$$

and $u$ is some fixed element in $H . \mathrm{Xu}[6]$ introduced the following iteration process:

$$
u_{k+1}=\left(I-\lambda_{k+1} A\right) T_{k+1} u_{k}+\lambda_{k+1} u,
$$

where $I$ is the identity mapping of $H$, and proved the following result.

Theorem 1.3 [6] Let conditions (L1), (L2) and (L3) or (L4) be satisfied. Assume in addition that (1.4) holds. Then the sequence $\left\{u_{k}\right\}$ generated by algorithm (1.7) converges strongly to the unique solution of (1.1) with $F x=A x-u$.

Very recently, Liu and Cui [8] showed that the condition

$$
C=\bigcap_{i=1}^{N} \operatorname{Fix}\left(T_{i}\right)=\operatorname{Fix}\left(T_{1} T_{2} \cdots T_{N}\right)
$$

is sufficient for (1.4) if $C \neq \emptyset$.

In this paper, we introduce a new algorithm based on a combination of the steepest descent method for variational inequalities with the Krasnoselskii-Mann method for fixed 
point problems to solve (1.1) with $C=\bigcap_{i=1}^{N} \operatorname{Fix}\left(T_{i}\right)$, where $T_{i}$ is a nonexpansive mapping on $H$ for each $i$.

Given a starting point $x_{1} \in H$, the iteration is defined by

$$
\left\{\begin{array}{l}
x_{1} \in H, \\
y_{k}^{0}=\left(I-\lambda_{k} \mu F\right) x_{k}, \\
y_{k}^{i}=\left(1-\beta_{k}^{i}\right) x_{k}+\beta_{k}^{i} T_{i} y_{k}^{i-1}, \quad i=1, \ldots, N, \\
x_{k+1}=y_{k}^{N},
\end{array}\right.
$$

and the sequences of parameters $\left\{\lambda_{k}\right\}$ and $\left\{\beta_{k}^{i}\right\} \subset(0,1)$ satisfy the following conditions:

$$
\left\{\begin{array}{l}
\lambda_{k} \rightarrow 0 \quad \text { as } k \rightarrow \infty \quad \text { and } \quad \sum \lambda_{k}=\infty ; \\
\beta_{k}^{i} \rightarrow \beta^{i}, \quad 0<\beta^{i}<1, i=1, \ldots, N-1 ; \\
0<\liminf _{k \rightarrow \infty} \beta_{k}^{N} \leq \lim \sup _{k \rightarrow \infty} \beta_{k}^{N}<1 .
\end{array}\right.
$$

In Section 2, we prove the strong convergence theorem for (1.9)-(1.10) without conditions (L3), (L4) and (1.8). An application to the case that $T_{i}$ is a $\gamma_{i}$-strictly pseudocontractive mapping is given in Section 3.

\section{Main results}

We need the following lemmas for the proof of our main result.

\section{Lemma 2.1 [9]}

(i) $\|x+y\|^{2} \leq\|x\|^{2}+2\langle y, x+y\rangle, \forall x, y \in H$.

(ii) $\|(1-t) x+t y\|^{2}=(1-t)\|x\|^{2}+t\|y\|^{2}-(1-t) t\|x-y\|^{2}, \forall x, y \in H$, and for any fixed $t \in[0,1]$.

From [4], we have the following lemma.

Lemma $2.2[4]\left\|T^{\lambda} x-T^{\lambda} y\right\| \leq(1-\lambda \tau)\|x-y\|, \forall x, y \in H$ and for a fixed number $\mu \in$ $\left(0,2 \eta / L^{2}\right), \lambda \in(0,1)$, where $\tau=1-\sqrt{1-\mu\left(2 \eta-\mu L^{2}\right)} \in(0,1)$ and $T^{\lambda} x=(I-\lambda \mu F) x$ for $x \in H$.

Lemma $2.3[10,11]$ Assume that $T$ is a nonexpansive self-map of a closed convex subset $K$ of a real Hilbert space $H$. If $T$ has a fixed point, then $I-T$ is demiclosed; that is, whenever $\left\{x_{k}\right\}$ is a sequence in $K$ weakly converging to some $x \in K$ and the sequence $\left\{(I-T) x_{k}\right\}$ strongly converges to some $y$, it follows that $(I-T) x=y$.

Lemma 2.4 [12] Let $\left\{x_{k}\right\}$ and $\left\{z_{k}\right\}$ be bounded sequences in a Banach space E such that

$$
x_{k+1}=\left(1-\beta_{k}\right) x_{k}+\beta_{k} z_{k}
$$

for $k \geq 1$, where $\left\{\beta_{k}\right\}$ is in $[0,1]$ such that

$$
0<\liminf _{k \rightarrow \infty} \beta_{k} \leq \limsup _{k \rightarrow \infty} \beta_{k}<1
$$


Assume that

$$
\limsup _{k \rightarrow \infty}\left\|z_{k+1}-z_{k}\right\|-\left\|x_{k+1}-x_{k}\right\| \leq 0
$$

Then $\lim _{k \rightarrow \infty}\left\|x_{k}-z_{k}\right\|=0$.

Lemma 2.5 [6] Let $\left\{a_{k}\right\}$ be a sequence of nonnegative real numbers satisfying the condition

$$
a_{k+1} \leq\left(1-b_{k}\right) a_{k}+b_{k} c_{k}
$$

where $\left\{b_{k}\right\}$ and $\left\{c_{k}\right\}$ are sequences of real numbers such that

(i) $b_{k} \in[0,1]$ and $\sum_{k=1}^{\infty} b_{k}=\infty$;

(ii) $\limsup _{k \rightarrow \infty} c_{k} \leq 0$.

Then $\lim _{k \rightarrow \infty} a_{k}=0$.

Now, we are in a position to prove the following main result.

Theorem 2.6 Let $H$ be a real Hilbert space, and let $F: H \rightarrow H$ be an L-Lipschitz continuous and $\eta$-strongly monotone mapping for some constants $L, \eta>0$. Let $\left\{T_{i}\right\}_{i=1}^{N}$ be $N$ nonexpansive self-maps of $H$ such that

$$
C=\bigcap_{i=1}^{N} \operatorname{Fix}\left(T_{i}\right) \neq \emptyset .
$$

Then the sequence $\left\{x_{k}\right\}$ defined by (1.9)-(1.10) converges strongly to the unique element $p^{*}$ in (1.1).

Proof First, we prove that $\left\{x_{k}\right\}$ is bounded. By Lemma 2.2, we have, for any $p \in C$, from (1.9) that

$$
\begin{aligned}
\left\|y_{k}^{0}-p\right\| & =\left\|\left(I-\lambda_{k} \mu F\right) x_{k}-p\right\|=\left\|\left(I-\lambda_{k} \mu F\right) x_{k}-\left(I-\lambda_{k} \mu F\right) p-\lambda_{k} \mu F(p)\right\| \\
& \leq\left(1-\lambda_{k} \tau\right)\left\|x_{k}-p\right\|+\lambda_{k} \mu\|F(p)\| .
\end{aligned}
$$

Put $M_{p}=\max \left\{\left\|x_{1}-p\right\|, \frac{\mu}{\tau}\|F(p)\|\right\}$. Then $\left\|x_{1}-p\right\| \leq M_{p}$. So, if $\left\|x_{k}-p\right\| \leq M_{p}$, then $\left\|y_{k}^{0}-p\right\| \leq$ $M_{p}$. This conclusion has a place for $\left\{y_{k}^{i}\right\}$ with $i=1, \ldots, N-1$. Indeed,

$$
\begin{aligned}
\left\|y_{k}^{i}-p\right\| & =\left\|\left(1-\beta_{k}^{i}\right)\left(x_{k}-p\right)+\beta_{k}^{i}\left(T_{i} y_{k}^{i-1}-T_{i} p\right)\right\| \\
& \leq\left(1-\beta_{k}^{i}\right)\left\|x_{k}-p\right\|+\beta_{k}^{i}\left\|y_{k}^{i-1}-p\right\| \\
& \leq\left(1-\beta_{k}^{i}\right) M_{p}+\beta_{k}^{i} M_{p} \\
& =M_{p} .
\end{aligned}
$$

Then

$$
\begin{aligned}
\left\|x_{k+1}-p\right\| & =\left\|\left(1-\beta_{k}^{N}\right)\left(x_{k}-p\right)+\beta_{k}^{N}\left(T_{i} y_{k}^{i-1}-T_{i} p\right)\right\| \\
& \leq\left(1-\beta_{k}^{N}\right)\left\|x_{k}-p\right\|+\beta_{k}^{i} N\left\|y_{k}^{N-1}-p\right\|
\end{aligned}
$$




$$
\begin{aligned}
& \leq\left(1-\beta_{k}^{i}\right) M_{p}+\beta_{k}^{i} M_{p} \\
& =M_{p} .
\end{aligned}
$$

Therefore, the sequence $\left\{x_{k}\right\}$ is bounded. So, the sequences $\left\{F\left(x_{k}\right)\right\},\left\{y_{k}^{i}\right\}$, and $\left\{T_{i} y_{k}^{i-1}\right\}(i=$ $1,2, \ldots, N)$ are also bounded. Without loss of generality, we assume that they are bounded by a positive constant $M_{1}$.

Let $z_{k}=T_{N} y_{k}^{N-1}$. Then we have from (1.9) that

$$
x_{k+1}=\left(1-\beta_{k}^{N}\right) x_{k}+\beta_{k}^{N} z_{k}
$$

and

$$
\begin{aligned}
\left\|z_{k+1}-z_{k}\right\|= & \left\|T_{N} y_{k+1}^{N-1}-T_{N} y_{k}^{N-1}\right\| \leq\left\|y_{k+1}^{N-1}-y_{k}^{N-1}\right\| \\
= & \|\left(1-\beta_{k+1}^{N-1}\right) x_{k+1}+\beta_{k+1}^{N-1} T_{N-1} y_{k+1}^{N-2} \\
& -\left[\left(1-\beta_{k}^{N-1}\right) x_{k}+\beta_{k}^{N-1} T_{N-1} y_{k}^{N-2}\right] \| \\
\leq & \left(1-\beta_{k+1}^{N-1}\right)\left\|x_{k+1}-x_{k}\right\|+2 M_{1}\left|\beta_{k+1}^{N-1}-\beta_{k}^{N-1}\right| \\
& +\beta_{k+1}^{N-1}\left\|T_{N-1} y_{k+1}^{N-2}-T_{N-1} y_{k}^{N-2}\right\| \\
\leq & \left(1-\beta_{k+1}^{N-1}\right)\left\|x_{k+1}-x_{k}\right\|+2 M_{1}\left|\beta_{k+1}^{N-1}-\beta_{k}^{N-1}\right| \\
& +\beta_{k+1}^{N-1}\left\|y_{k+1}^{N-2}-y_{k}^{N-2}\right\| \\
\leq & \left(1-\beta_{k+1}^{N-1}\right)\left\|x_{k+1}-x_{k}\right\|+2 M_{1}\left|\beta_{k+1}^{N-1}-\beta_{k}^{N-1}\right| \\
& +\beta_{k+1}^{N-1}\left[\left(1-\beta_{k+1}^{N-2}\right)\left\|x_{k+1}-x_{k}\right\|+2 M_{1}\left|\beta_{k+1}^{N-2}-\beta_{k}^{N-2}\right|\right. \\
& \left.+\beta_{k+1}^{N-2}\left\|y_{k+1}^{N-3}-y_{k}^{N-3}\right\|\right] \\
\leq & \left(1-\beta_{k+1}^{N-1} \beta_{k+1}^{N-2}\right)\left\|x_{k+1}-x_{k}\right\|+2 M_{1}\left(\left|\beta_{k+1}^{N-1}-\beta_{k}^{N-1}\right|\right. \\
& \left.+\left|\beta_{k+1}^{N-2}-\beta_{k}^{N-2}\right|\right)+\beta_{k+1}^{N-1} \beta_{k+1}^{N-2}\left\|y_{k+1}^{N-3}-y_{k}^{N-3}\right\| \\
\leq & \cdots \\
\leq & \left(1-\prod_{i=1}^{N-1} \beta_{k+1}^{i}\right)\left\|x_{k+1}-x_{k}\right\|+\prod_{i=1}^{N-1} \beta_{k+1}^{i}\left\|y_{k+1}^{0}-y_{k}^{0}\right\| \\
& +2 M_{1} \sum_{i=1}^{N-1}\left|\beta_{k+1}^{i}-\beta_{k}^{i}\right| . \\
&
\end{aligned}
$$

On the other hand,

$$
\begin{aligned}
\left\|y_{k+1}^{0}-y_{k}^{0}\right\| & =\left\|\left(I-\lambda_{k+1} \mu F\right) x_{k+1}-\left(I-\lambda_{k} \mu F\right) x_{k}\right\| \\
& \leq\left\|x_{k+1}-x_{k}\right\|+M_{1}\left(\lambda_{k+1}+\lambda_{k}\right) .
\end{aligned}
$$

So, we obtain that

$$
\left\|z_{k+1}-z_{k}\right\|-\left\|x_{k+1}-x_{k}\right\| \leq M_{1}\left(\lambda_{k+1}+\lambda_{k}\right) \prod_{i=1}^{N-1} \beta_{k+1}^{i}+2 M_{1} \sum_{i=1}^{N-1}\left|\beta_{k+1}^{i}-\beta_{k}^{i}\right| .
$$


Since $\lambda_{k} \rightarrow 0$ and $\beta_{k}^{i} \rightarrow \beta^{i}, i=1, \ldots, N-1$, we have

$$
\limsup _{k \rightarrow \infty}\left\|z_{k+1}-z_{k}\right\|-\left\|x_{k+1}-x_{k}\right\| \leq 0
$$

By Lemma 2.4, $\left\|x_{k}-z_{k}\right\| \rightarrow 0$ as $k \rightarrow \infty$, i.e., $\left\|x_{k}-T_{N} y_{k}^{N-1}\right\| \rightarrow 0$.

Now, we prove that $\left\|x_{k}-T_{i} y_{k}^{i-1}\right\| \rightarrow 0$ for $i=1, \ldots, N-1$. First, we prove $\| x_{k}-$ $T_{N-1} y_{k}^{N-2} \| \rightarrow 0$. Let $\left\{x_{k_{n}}\right\}$ be a subsequence of $\left\{x_{k}\right\}$ such that

$$
\limsup _{k \rightarrow \infty}\left\|x_{k}-T_{N-1} y_{k}^{N-2}\right\|=\lim _{n \rightarrow \infty}\left\|x_{k_{n}}-T_{N-1} y_{k_{n}}^{N-2}\right\|
$$

and let $\left\{x_{k_{j}}\right\}$ be a subsequence of $\left\{x_{k_{n}}\right\}$ such that

$$
\limsup _{n \rightarrow \infty}\left\|x_{k_{n}}-p\right\|=\lim _{j \rightarrow \infty}\left\|x_{k_{j}}-p\right\|
$$

Further,

$$
\begin{aligned}
\left\|x_{k_{j}}-p\right\| \leq & \left\|x_{k_{j}}-T_{N} y_{k_{j}}^{N-1}\right\|+\left\|T_{N} y_{k_{j}}^{N-1}-T_{N} p\right\| \\
\leq & \left\|x_{k_{j}}-T_{N} y_{k_{j}}^{N-1}\right\|+\left\|y_{k_{j}}^{N-1}-p\right\| \\
\leq & \left\|x_{k_{j}}-T_{N} y_{k_{j}}^{N-1}\right\|+\left(1-\beta_{k}^{N-1}\right)\left\|x_{k_{j}}-p\right\| \\
& +\beta_{k_{j}}^{N-1}\left\|T_{N-1} y_{k_{j}}^{N-2}-T_{N-1} p\right\| \\
\leq & \left\|x_{k_{j}}-T_{N} y_{k_{j}}^{N-1}\right\|+\left(1-\beta_{k}^{N-1}\right)\left\|x_{k_{j}}-p\right\|+\beta_{k_{j}}^{N-1}\left\|y_{k_{j}}^{N-2}-p\right\| \| \\
\leq & \left\|x_{k_{j}}-T_{N} y_{k_{j}}^{N-1}\right\|+\left(1-\beta_{k}^{N-1}\right)\left\|x_{k_{j}}-p\right\| \\
& +\beta_{k_{j}}^{N-1}\left[\left(1-\beta_{k}^{N-2}\right)\left\|x_{k_{j}}-p\right\|+\beta_{k_{j}}^{N-2}\left\|T_{N-2} y_{k_{j}}^{N-3}-T_{N-2} p\right\|\right] \\
\leq & \left\|x_{k_{j}}-T_{N} y_{k_{j}}^{N-1}\right\|+\left(1-\beta_{k}^{N-1} \beta_{k_{j}}^{N-2}\right)\left\|x_{k_{j}}-p\right\| \\
& +\beta_{k_{j}}^{N-1} \beta_{k_{j}}^{N-2}\left\|y_{k_{j}}^{N-2}-p\right\| \\
\leq & \ldots \\
\leq & \left\|x_{k_{j}}-T_{N} y_{k_{j}}^{N-1}\right\|+\left(1-\prod_{i=1}^{N-1} \beta_{k_{j}}^{i}\right)\left\|x_{k_{j}}-p\right\|+\prod_{i=1}^{N-1} \beta_{k_{j}}^{i}\left\|y_{k_{j}}^{0}-p\right\| .
\end{aligned}
$$

Since

$$
\left\|y_{k_{j}}^{0}-p\right\| \leq\left(1-\lambda_{k_{j}} \tau\right)\left\|x_{k_{j}}-p\right\|+\lambda_{k_{j}} \mu\|F(p)\|,
$$

we have

$$
\begin{aligned}
\left\|x_{k_{j}}-p\right\| & \leq\left\|x_{k_{j}}-T_{N} y_{k_{j}}^{N-1}\right\|+\left\|y_{k_{j}}^{N-1}-p\right\| \\
& \leq\left\|x_{k_{j}}-T_{N} y_{k_{j}}^{N-1}\right\|+\left\|x_{k_{j}}-p\right\|+\prod_{i=1}^{N-1} \beta_{k_{j}}^{i} \lambda_{k_{j}} \mu\|F(p)\| .
\end{aligned}
$$


Therefore,

$$
\lim _{j \rightarrow \infty}\left\|x_{k_{j}}-p\right\|=\lim _{j \rightarrow \infty}\left\|y_{k_{j}}^{N-1}-p\right\|
$$

Next, by Lemma 2.1 we have

$$
\begin{aligned}
& \left\|y_{k_{j}}^{N-1}-p\right\|^{2}=\left(1-\beta_{k_{j}}^{N-1}\right)\left\|x_{k_{j}}-p\right\|^{2}+\beta_{k_{j}}^{N-1}\left\|T_{N-1} y_{k_{j}}^{N-2}-p\right\|^{2} \\
& -\left(1-\beta_{k_{j}}^{N-1}\right) \beta_{k_{j}}^{N-1}\left\|x_{k_{j}}-T_{N-1} y_{k_{j}}^{N-2}\right\|^{2} \\
& \leq\left(1-\beta_{k_{j}}^{N-1}\right)\left\|x_{k_{j}}-p\right\|^{2}+\beta_{k_{j}}^{N-1}\left\|y_{k_{j}}^{N-2}-p\right\|^{2} \\
& -\left(1-\beta_{k_{j}}^{N-1}\right) \beta_{k_{j}}^{N-1}\left\|x_{k_{j}}-T_{N-1} y_{k_{j}}^{N-2}\right\|^{2} \\
& \leq\left(1-\beta_{k_{j}}^{N-1}\right)\left\|x_{k_{j}}-p\right\|^{2} \\
& +\beta_{k_{j}}^{N-1}\left\|\left(1-\beta_{k_{j}}^{N-2}\right)\left(x_{k_{j}}-p\right)+\beta_{k_{j}}^{N-2}\left(T_{N-2} y_{k_{j}}^{N-3}-p\right)\right\|^{2} \\
& -\left(1-\beta_{k_{j}}^{N-1}\right) \beta_{k_{j}}^{N-1}\left\|x_{k_{j}}-T_{N-1} y_{k_{j}}^{N-2}\right\|^{2} \\
& \leq\left(1-\beta_{k_{j}}^{N-1} \beta_{k_{j}}^{N-2}\right)\left\|x_{k_{j}}-p\right\|^{2}+\beta_{k_{j}}^{N-1} \beta_{k_{j}}^{N-2}\left\|y_{k_{j}}^{N-3}-p\right\|^{2} \\
& -\left(1-\beta_{k_{j}}^{N-1}\right) \beta_{k_{j}}^{N-1}\left\|x_{k_{j}}-T_{N-1} y_{k_{j}}^{N-2}\right\|^{2} \\
& \leq \cdots \\
& \leq\left(1-\prod_{i=1}^{N-1} \beta_{k_{j}}^{i}\right)\left\|x_{k_{j}}-p\right\|^{2}+\prod_{i=1}^{N-1} \beta_{k_{j}}^{i}\left\|y_{k_{j}}^{0}-p\right\|^{2} \\
& -\left(1-\beta_{k_{j}}^{N-1}\right) \beta_{k_{j}}^{N-1}\left\|x_{k_{j}}-T_{N-1} y_{k_{j}}^{N-2}\right\|^{2} .
\end{aligned}
$$

On the other hand, by Lemma 2.1 we get

$$
\begin{aligned}
\left\|y_{k}^{0}-p\right\|^{2} & =\left\|\left(I-\lambda_{k} \mu F\right) x_{k}-p\right\|^{2} \\
& =\left\|x_{k}-p-\left(I-\lambda_{k} \mu F\right) x_{k}\right\| \\
& \leq\left\|x_{k}-p\right\|^{2}-2 \lambda_{k} \mu\left\langle F\left(x_{k}\right), x_{k}-p\right\rangle \\
& \leq\left\|x_{k}-p\right\|^{2}+2 \lambda_{k} \mu M_{1} M_{p} .
\end{aligned}
$$

Without loss of generality, assume that $\alpha \leq \beta_{k}^{i} \leq \beta, k \geq 1$ for $i=1, \ldots, N-1$ and some $\alpha, \beta \in(0,1)$. Then we obtain that

$$
\alpha(1-\beta)\left\|x_{k_{j}}-T_{N-1} y_{k_{j}}^{N-2}\right\|^{2} \leq\left\|x_{k_{j}}-p\right\|^{2}-\left\|y_{k_{j}}^{N-1}-p\right\|^{2}+2 \lambda_{k_{j}} \mu M_{1} M_{p} \prod_{i=1}^{N-1} \beta_{k_{j}}^{i},
$$

which with $\lambda_{k} \rightarrow 0$ and (2.1) implies that $\left\|x_{k_{j}}-T_{N-1} y_{k_{j}}^{N-2}\right\| \rightarrow 0$ as $j \rightarrow \infty$. So, $\| x_{k}-$ $T_{N-1} y_{k}^{N-2} \| \rightarrow 0$ as $k \rightarrow \infty$.

Similarly, we obtain that $\left\|x_{k}-T_{N-2} y_{k}^{N-3}\right\| \rightarrow 0, \ldots,\left\|x_{k}-T_{1} y_{k}^{0}\right\| \rightarrow 0$ as $k \rightarrow \infty$.

Further, we prove that $\left\|x_{k}-T_{i} x_{k}\right\| \rightarrow 0$ as $k \rightarrow \infty$ for $i=1, \ldots, N$. First, note that $\| y_{k}^{0}-$ $x_{k}\left\|=\lambda_{k}\right\| F\left(x_{k}\right) \| \rightarrow 0$ as $k \rightarrow \infty$ because $\lambda_{k} \rightarrow 0$ and $\left\|F\left(x_{k}\right)\right\| \leq M_{1}$, and $\left\|y_{k}^{i}-x_{k}\right\|=\beta_{k}^{i} \| x_{k}-$ 
$T_{i} y_{k}^{i-1} \| \rightarrow 0$ because $\left\|x_{k}-T_{i} y_{k}^{i-1}\right\| \rightarrow 0$, for $i=1, \ldots, N$. Now, from

$$
\begin{aligned}
\left\|x_{k}-T_{i} x_{k}\right\| & \leq\left\|x_{k}-T_{i} y_{k}^{i-1}\right\|+\left\|T_{i} y_{k}^{i-1}-T_{i} x_{k}\right\| \\
& \leq\left\|x_{k}-T_{i} y_{k}^{i-1}\right\|+\left\|y_{k}^{i-1}-x_{k}\right\|
\end{aligned}
$$

and $\left\|x_{k}-T_{i} y_{k}^{i-1}\right\|,\left\|y_{k}^{i-1}-x_{k}\right\| \rightarrow 0$, it follows that $\left\|x_{k}-T_{i} x_{k}\right\| \rightarrow 0$ for $i=1, \ldots, N$.

Further, we have

$$
\limsup _{k \rightarrow \infty}\left\langle F\left(p^{*}\right), p^{*}-x_{k}\right\rangle \leq 0
$$

Indeed, let $\left\{x_{k_{j}}\right\}$ be a subsequence of $\left\{x_{k}\right\}$ that converges weakly to $\tilde{p}$ such that

$$
\limsup _{k \rightarrow \infty}\left\langle F\left(p^{*}\right), p^{*}-x_{k}\right\rangle=\lim _{j \rightarrow \infty}\left\langle F\left(p^{*}\right), p^{*}-x_{k_{j}}\right\rangle .
$$

Then $\left\|x_{k_{j}}-T_{i} x_{k_{j}}\right\| \rightarrow 0$. So, by Lemma 2.3, $\tilde{p} \in C$. Therefore, from (1.1) it implies (2.2).

Finally, we estimate the value

$$
\begin{aligned}
\left\|x_{k+1}-p^{*}\right\|^{2} \leq & \left(1-\beta_{k}^{N}\right)\left\|x_{k}-p^{*}\right\|^{2}+\beta_{k}^{N}\left\|T_{N} y_{k}^{N-1}-T_{N} p^{*}\right\|^{2} \\
\leq & \left(1-\beta_{k}^{N}\right)\left\|x_{k}-p^{*}\right\|^{2}+\beta_{k}^{N}\left\|y_{k}^{N-1}-T_{N} p^{*}\right\|^{2} \\
\leq & \left(1-\beta_{k}^{N}\right)\left\|x_{k}-p^{*}\right\|^{2} \\
& +\beta_{k}^{N}\left[\left(1-\beta_{k}^{N-1}\right)\left\|x_{k}-p^{*}\right\|^{2}+\beta_{k}^{N-1}\left\|T_{N-1} y_{k}^{N-2}-T_{N-1} p^{*}\right\|^{2}\right] \\
\leq & \left(1-\beta_{k}^{N} \beta_{k}^{N-1}\right)\left\|x_{k}-p^{*}\right\|^{2}+\beta_{k}^{N} \beta_{k}^{N-1}\left\|y_{k}^{N-1}-T_{N} p^{*}\right\|^{2} \\
\leq & \left(1-\beta_{k}^{N} \beta_{k}^{N-1}\right)\left\|x_{k}-p^{*}\right\|^{2}+\beta_{k}^{N} \beta_{k}^{N-1}\left[\left(1-\beta_{k}^{N-2}\right)\left\|x_{k}-p^{*}\right\|^{2}\right. \\
& \left.+\beta_{k}^{N-2}\left\|T_{N-2} y_{k}^{N-3}-T_{N-2} p^{*}\right\|^{2}\right] \\
& \leq \cdots \\
\leq & \left(1-\prod_{i=1}^{N} \beta_{k}^{i}\right)\left\|x_{k}-p^{*}\right\|^{2}+\prod_{i=1}^{N} \beta_{k}^{i}\left\|y_{k}^{0}-p^{*}\right\|^{2} .
\end{aligned}
$$

On the other hand, since

$$
\begin{aligned}
\left\|y_{k}^{0}-p^{*}\right\|^{2} & =\left\|\left(I-\lambda_{k} \mu F\right) x_{k}-p^{*}\right\|^{2} \\
& =\left\|\left(I-\lambda_{k} \mu F\right)\left(x_{k}-p^{*}\right)-\lambda_{k} \mu F\left(p^{*}\right)\right\|^{2} \\
& \leq\left(1-\lambda_{k} \tau\right)\left\|x_{k}-p^{*}\right\|^{2}-2 \lambda_{k} \mu\left\langle F\left(x_{k}\right), y_{k}^{0}-p^{*}\right\rangle,
\end{aligned}
$$

we have

$$
\begin{aligned}
\left\|x_{k+1}-p^{*}\right\|^{2} \leq & \left(1-\prod_{i=1}^{N} \beta_{k}^{i}\right)\left\|x_{k}-p^{*}\right\|^{2}+\prod_{i=1}^{N} \beta_{k}^{i}\left(1-\lambda_{k} \tau\right)\left\|x_{k}-p^{*}\right\|^{2} \\
& +\left[2 \lambda_{k} \mu\left\langle F\left(p^{*}\right), p^{*}-x_{k}\right\rangle+2 \lambda_{k} \mu\left\langle F\left(p^{*}\right), x_{k}-y_{k}^{0}\right\rangle\right] \prod_{i=1}^{N} \beta_{k}^{i}
\end{aligned}
$$




$$
\begin{aligned}
\leq & \left(1-\lambda_{k} \tau \prod_{i=1}^{N} \beta_{k}^{i}\right)\left\|x_{k}-p^{*}\right\|^{2} \\
& +\lambda_{k} \tau \prod_{i=1}^{N} \beta_{k}^{i}\left[\frac{2 \mu}{\tau}\left\langle F\left(p^{*}\right), p^{*}-x_{k}\right\rangle+\frac{2 \mu}{\tau}\left\langle F\left(p^{*}\right), x_{k}-y_{k}^{0}\right\rangle\right] .
\end{aligned}
$$

Using Lemma 2.5 with

$$
\begin{aligned}
& a_{k}=\left\|x_{k}-p^{*}\right\|, \\
& b_{k}=\lambda_{k} \tau \prod_{i=1}^{N} \beta_{k}^{i}, \\
& c_{k}=\frac{2 \mu}{\tau}\left\langle F\left(p^{*}\right), p^{*}-x_{k}\right\rangle+\frac{2 \mu}{\tau}\left\langle F\left(p^{*}\right), x_{k}-y_{k}^{0}\right\rangle,
\end{aligned}
$$

$\left\|x_{k}-y_{k}^{0}\right\| \rightarrow 0$ and (2.2), we have that $\left\|x_{k}-p^{*}\right\| \rightarrow 0$. This completes the proof.

\section{Application}

Recall that a mapping $S: H \rightarrow H$ is called $\gamma$-strictly pseudocontractive if there exists a constant $\gamma \in[0,1)$ such that

$$
\|S x-S y\|^{2} \leq\|x-y\|^{2}+\gamma\|(I-S) x-(I-S) y\|^{2}, \quad \forall x, y \in H .
$$

It is well known [9] that a mapping $T: H \rightarrow H$ by $T x=\alpha x+(1-\alpha) S x$ with a fixed $\alpha \in[\gamma, 1)$ for all $x \in H$ is a nonexpansive mapping and $\operatorname{Fix}(T)=\operatorname{Fix}(S)$.

Using this fact, we can extend our result to the case $C=\bigcap_{i=1}^{N} \operatorname{Fix}\left(S_{i}\right)$, where $S_{i}$ is $\gamma_{i}$-strictly pseudocontractive as follows.

Let $\alpha_{i} \in\left[\gamma_{i}, 1\right)$ be fixed numbers. Then $C=\bigcap_{i=1}^{N} \operatorname{Fix}\left(\tilde{T}_{i}\right)$ with

$$
\tilde{T}_{i} y=\alpha_{i} y+\left(1-\alpha_{i}\right) S_{i} y,
$$

a nonexpansive mapping, for each $i=1, \ldots, N$. So, we have the following result.

Theorem 3.1 Let $H$ be a real Hilbert space, and let $F: H \rightarrow H$ be an L-Lipschitzian and $\eta$-strongly monotone mapping for some constants $L, \eta>0$. Let $\left\{S_{i}\right\}_{i=1}^{N}$ be $N \gamma_{i}$-strictly pseudocontractive self-maps of $H$ such that

$$
C=\bigcap_{i=1}^{N} \operatorname{Fix}\left(S_{i}\right) \neq \emptyset .
$$

Let $\alpha_{i} \in\left[\gamma_{i}, 1\right), \mu \in\left(0,2 \eta / L^{2}\right)$. Assume that $\left\{\lambda_{k}\right\},\left\{\beta_{k}^{i}\right\} \subset(0,1)$ satisfy (1.10). Then the sequence $\left\{x_{k}\right\}$ defined by (1.9) with $T_{i}$ replaced by $\tilde{T}_{i}$ of (3.1) converges strongly to the unique element $p^{*}$ of (1.1).

\section{Numerical example}

Consider the following optimization problem: find an element

$$
p^{*} \in C: \varphi\left(p^{*}\right)=\min _{C} \varphi(x)
$$


Table 1 Iterations of scheme (1.9), where starting point $x^{0}=(4 ; 15)$

\begin{aligned} & \hline $\boldsymbol{k}$ th $\boldsymbol{x}_{\boldsymbol{k}}$ by algorithm (1.9) \\ & \hline 0$(4.00000 ; 15.00000) \\ & 10(4.10755 ; 13.434117) \\ & 20(3.89572 ; 12.58288) \\ & 1,000(1.52757 ; 3.110295) \\ & 1,500(1.28179 ; 2.12718) \\ & 2,000(1.13269 ; 1.53076) \\ & 2,500(1.03367 ; 1.13468) \\ & 3,000(0.99933 ; 0.99738) \\ & 3,500(0.99938 ; 0.99517) \\ & 4,000(0.99942 ; 0.99768) \\ & 4,500(0.99945 ; 0.99781) \\ & 5,000(0.99948 ; 0.99792) \\ &$\hline\end{aligned}

where $\varphi(x)=\|x\|^{2} / 2, x=\left(x_{1}, x_{2}\right) \in \mathbf{E}^{2}$, Euclid space, and $C=C_{1} \cap C_{2}$, defined by

$$
\begin{aligned}
& C_{1}=\left\{\left(x_{1}, x_{2}\right) \in \mathbf{E}^{2}: x_{1}-2 x_{2}+1 \leq 0\right\}, \\
& C_{2}=\left\{\left(x_{1}, x_{2}\right) \in \mathbf{E}^{2}: 4 x_{1}-x_{2}-3 \geq 0\right\} .
\end{aligned}
$$

Clearly, the above problem possesses a unique solution $p^{*}=(1 ; 1)$ and $F$, the Fréchet derivative of $\varphi$, is 1 -Lipschitz continuous and (1/2)-strongly monotone. Starting with the point $x^{0}=\left(x_{1}^{0} ; x_{2}^{0}\right)=(4 ; 15), \mu=1 / 10 \in\left(0 ; 2 \eta / L^{2}\right)$ and $\lambda_{k}=(k+1)^{1 / 2}$, we obtained the result in Table 1.

\section{Competing interests}

The authors declare that they have no competing interests.

\section{Authors' contributions}

The main idea of this paper was proposed by JKK. JKK and NB prepared the manuscript initially and performed all the steps of proof in this research. All authors read and approved the final manuscript.

\section{Author details}

${ }^{1}$ Department of Mathematics Education, Kyungnam University, Changwon, 631-701, Korea. ${ }^{2}$ Vietnamese Academy of Science and Technology, Institute of Information Technology, 18, Hoang Quoc Viet, Cau Giay, Hanoi, Vietnam.

\section{Acknowledgements}

This work was supported by Basic Science Research Program through the National Research Foundation of Korea (NRF) funded by the Ministry of Education, Science and Technology (2012R1A1A2042138).

\section{Received: 8 April 2013 Accepted: 18 August 2013 Published: 3 September 2013}

\section{References}

1. Kinderlehrer, D, Stampacchia, G: An Introduction to Variational Inequalities and Their Applications. Academic Press, New York (1980)

2. Glowinski, R: Numerical Methods for Nonlinear Variational Problems. Springer, New York (1984)

3. Zeidler, E: Nonlinear Functional Analysis and Its Applications. Springer, New York (1985)

4. Yamada, Y: The hybrid steepest-descent method for variational inequalities problems over the intersection of the fixed point sets of nonexpansive mappings. In: Butnariu, D, Censor, Y, Reich, S (eds.) Inherently Parallel Algorithms in Feasibility and Optimization and Their Applications, pp. 473-504. North-Holland, Amsterdam (2001)

5. Zeng, LC, Yao, JC: Implicit iteration scheme with perturbed mapping for common fixed points of a finite family of nonexpansive mappings. Nonlinear Anal. TMA 64, 2507-2515 (2006)

6. Xu, HK: An iterative approach to quadratic optimization. J. Optim. Theory Appl. 116, 659-678 (2003)

7. Zeng, LC, Wong, NC, Yao, JC: Convergence analysis of modified hybrid steepest-descent methods with variable parameters for variational inequalities. J. Optim. Theory Appl. 132, 51-69 (2007)

8. Liu, X, Cui, Y: The common minimal-norm fixed point of a finite family of nonexpansive mappings. Nonlinear Anal. TMA 73, 76-83 (2010)

9. Marino, G, Xu, HK: Weak and strong convergence theorems for strict pseudo-contractions in Hilbert spaces. J. Math Anal. Appl. 329, 336-346 (2007)

10. Goebel, K, Kirk, WA: Topics in Metric Fixed Point Theory. Cambridge Studies in Advanced Math., vol. 28. Cambridge University Press, Cambridge (1990) 
11. Li, G, Kim, JK: Demiclosedness principle and asymptotic behavior for nonexpansive mappings in metric spaces. Appl. Math. Lett. 14(5), 645-649 (2001)

12. Suzuki, T: Strong convergence of approximated sequences for nonexpansive mappings in Banach spaces. Proc. Am. Math. Soc. 135, 99-106 (2007)

doi:10.1186/1029-242X-2013-419

Cite this article as: Kim and Buong: A new explicit iteration method for variational inequalities on the set of common fixed points for a finite family of nonexpansive mappings. Journal of Inequalities and Applications 2013 2013:419.

Submit your manuscript to a SpringerOpen ${ }^{\circ}$ journal and benefit from:

- Convenient online submission

- Rigorous peer review

Immediate publication on acceptance

Open access: articles freely available online

- High visibility within the field

- Retaining the copyright to your article

Submit your next manuscript at $\gg$ springeropen.com 